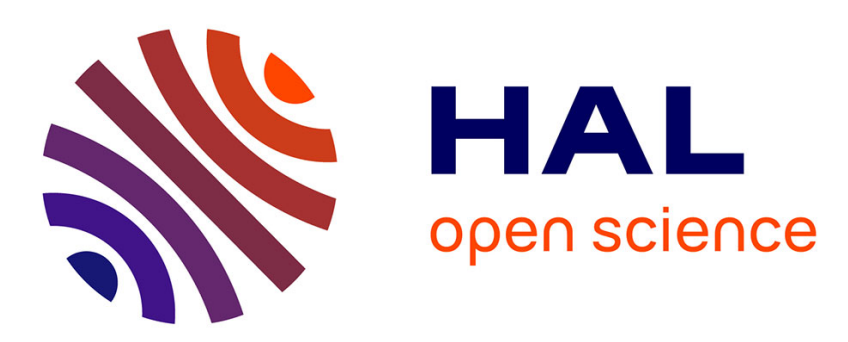

\title{
Jardin thérapeutique et démences : le dialogue comme unification des fonctions psychologiques
}

Manon Yzoard, Martine Batt, Alain Trognon, F. Verhaegen, Christel Jacob, Alina Pop, Louise Bernez, Reinhard Fescharek, Thérèse Rivasseau-Jonveaux

\section{- To cite this version:}

Manon Yzoard, Martine Batt, Alain Trognon, F. Verhaegen, Christel Jacob, et al.. Jardin thérapeutique et démences : le dialogue comme unification des fonctions psychologiques. NPG: Neurologie Psychiatrie - Gériatrie, 2017, 17 (100), pp.253 - 263. 10.1016/j.npg.2016.07.002 . hal-01938900

\section{HAL Id: hal-01938900 https://hal.science/hal-01938900}

Submitted on 29 Nov 2018

HAL is a multi-disciplinary open access archive for the deposit and dissemination of scientific research documents, whether they are published or not. The documents may come from teaching and research institutions in France or abroad, or from public or private research centers.
L'archive ouverte pluridisciplinaire HAL, est destinée au dépôt et à la diffusion de documents scientifiques de niveau recherche, publiés ou non, émanant des établissements d'enseignement et de recherche français ou étrangers, des laboratoires publics ou privés. 


\section{Jardin thérapeutique et démences : le dialogue comme unification des fonctions psychologiques}

Healing garden and dementia: dialogue as unification of psychological functions

Yzoard, M. ${ }^{\text {a* }}$, Batt, M. ${ }^{\mathrm{a}}$, Trognon, A. ${ }^{\mathrm{a}}$, Verhaegen, F. ${ }^{\mathrm{b}}$, Jacob, C. ${ }^{\mathrm{a}, \mathrm{c}}$, Pop, A. ${ }^{\mathrm{c}}$, Bernez, L. ${ }^{\mathrm{a}}$, Fescharek, R. ${ }^{\text {d }}$, Rivasseau-Jonveaux, T. ${ }^{\text {a, c, e }}$

a Groupe de Recherche sur les Communications (GRC), Laboratoire InterPsy, EA 4432, Université de lorraine, F54015 Nancy, France

b Laboratoire APEMAC/EPSaM, EA 4360, Université de Lorraine, 54015 Nancy, France

${ }^{\mathrm{c}}$ Centre Paul Spillmann, Centre hospitalier régional universitaire (CHRU) de Nancy, 54000 Nancy, France

${ }^{\mathrm{d}}$ Fescharek sculpture and design, Marburg Lahn, Germany

e Centre mémoire de ressources et de recherche (CMRR) de Lorraine, Centre hospitalier régional universitaire (CHRU) de Nancy, Hôpital de Brabois, 54500 Vandouvre-lès-Nancy, France

* Correspondance :

manon.yzoard@univ-lorraine.fr (M. Yzoard)

\section{Résumé :}

L'originalité de cet article réside dans l'expérimentation d'un jardin thérapeutique aménagé de références régionales naturelles et culturelles. Six patients présentant une démence ont été suivis dans leur promenade accompagnée. Les échanges entre patients et accompagnant (psychologue) mettent en évidence que l'interaction mobilise un système intégré de fonctions psychologiques gouverné par la fonction communicationnelle. Perception, émotion, mémoire, langage, planification, intelligence sociale sont sollicités par la rencontre avec végétaux et œuvres d'art, soulignant ainsi le rôle étayant de l'aménagement de l'environnement sur la cognition et les émotions des patients.

Mots-clés :

Maladie d'Alzheimer ; démences ; jardins thérapeutiques ; art ; interaction

Yzoard, M., Batt, M., Trognon, A., Verhaegen, F., Jacob, C., Pop, A., Bernez, L., Fescharek, R. \& RivasseauJonveaux, T. (2017). Jardin thérapeutique et démences : le dialogue comme unification des fonctions psychologiques. Neurologie - $\quad$ Psychiatrie - $\quad$ Gériatrie, 17(100), 253-263. https://doi.org/10.1016/j.npg.2016.07.002 


\section{Summary :}

The originality of this article lies in the experimentation of a healing garden embedded with natural and cultural regional references. Six patients with dementia were followed during their walk accompanied by a psychologist. The exchanges between patients and psychologist highlight that this interaction mobilizes an integrated system of psychological functions, a system governed by the communicative function. Perception, emotion, memory, language, planning, social intelligence are all solicited by the encounter with plants and artworks, thus underscoring the supporting role of the environmental design on the cognition and emotions of patients.

Keywords :

Alzheimer's disease ; dementia ; healing gardens ; artworks ; interaction

Les patients atteints de maladie d'Alzheimer (MA) subissent un déclin progressif de leurs fonctions cognitives au cours de l'évolution de la maladie. Le symptôme inaugural de la MA est marqué par une altération de la mémoire épisodique [1]. La mémoire sémantique et la mémoire de travail sont deux autres systèmes de mémoire de haut niveau qui peuvent être également perturbés au cours de la MA [1]. Ces troubles, qui ne sont pas exclusifs car d'autres fonctions cognitives sont touchées au cours de l'évolution de la maladie [1] ainsi que des troubles du comportement, vont être à l'origine d'une altération significative du fonctionnement social ou professionnel [2]. Ce déclin progressif affecte ainsi de façon intégrative la sphère cognitive, psycho-affective et sociale de l'individu [1].

La maladie d'Alzheimer et les maladies apparentées (MAMA) sont une préoccupation de santé publique comme le démontre la mise en place du nouveau Plan maladies neurodégénératives 2014-2019 [3] qui succède au Plan Alzheimer 2008-2012 [4]. Ce dernier, recommandait la mise à disposition de jardins thérapeutiques dans les unités spécialisées dans l'accueil de ces malades. Cette exhortation s'appuie sur un grand nombre de travaux qui établissent les effets bénéfiques des jardins thérapeutiques pour les patients atteints de MA, notamment pour les troubles des conduites élémentaires (appétit et sommeil) et pour les troubles psycho-comportementaux (agressivité, agitation, déambulation) [5-8] qui pourraient être ainsi réduits par l'utilisation d'environnements extérieurs adaptés et par des interventions psychosociales [9-10] comme celles médiatisées par l'art.

Soigner par l'activité artistique ou par l'exposition à des œuvres artistiques est une idée très ancienne dont on trouve l'origine dans la philosophie antique [11]. Aujourd'hui, l'art-thérapie correspond à des pratiques psychothérapeutiques dynamiques fondées sur la réalisation artistique en convoquant le corps et l'esprit lors d'ateliers créatifs [11]. En dehors de cette activité de production artistique, la simple perception d'une œuvre d'art va placer le spectateur dans une situation très active comme le souligne Changeux $[12,13]$ : d'abord, car l'œuvre d'art est un support public de communication intersubjective entre l'artiste et le spectateur. Mais aussi car les émotions, la mémoire, les représentations, les jugements et l'imagination sont sollicités lors de la contemplation d'une œuvre d'art. Devant cette dernière, 
le spectateur va être sensible à son efficacité esthétique qui va susciter l'activation de représentations en particulier visuelles, engageant ses émotions et sa raison afin de créer une représentation consciente de l'œuvre. Changeux [13] évoque également les processus mnémoniques engagés depuis cette perception. La contemplation d'œuvre d'art est codée de manière génétique et épigénétique chez l'homme. La rencontre de l'art va solliciter non seulement notre empreinte culturelle, mais va également activer nos mémoires inconscientes stockées à la suite de notre expérience personnelle.

La contemplation d'œuvres d'art, va être source d'émotions et va donc éveiller chez le spectateur différentes facultés cognitives [12-14]. Il semble important de connaître celles qui sont mobilisées dans ce contexte de fragilisation par la MAMA.

C'est l'objet de la recherche présentée ici, laquelle s'inscrit dans le champ de la psychologie sociale interactive des pathologies mentales. La communication inter-individuelle dialoguée y est étudiée en tant que processus dynamique complexe où se déploient simultanément différents processus mentaux : capacités cognitives, processus émotionnels, expression des fonctions mentales supérieures et effets (inter)subjectifs et sociaux [15]. Ces fonctions sont ainsi sollicitées tout naturellement par le dialogue qui matérialise les processus mentaux qui lient le sujet à son environnement.

Le jardin « art, mémoire et vie » du Centre Paul Spillmann du CHRU de Nancy vise à pallier les troubles cognitifs des patients atteints de MAMA, ainsi qu'à favoriser leur autonomie et leur bien-être. Sa conception conjugue une approche neuropsychologique et artistique [16, 17]. Celle-ci, confiée à un sculpteur qui est médecin de formation, repose sur la présence de sculptures et œuvres d'art conçues spécifiquement pour être vues et touchées mais également pour susciter des émotions propices aux réminiscences. Ces œuvres sont variées en terme de forme d'art (figuratif ou abstrait) et de représentations (mimétiques, stylisées ou nonobjectives) (figure 1). Les formes d'art figuratif représentent des objets du réel de façon mimétique ou stylisée : les représentations mimétiques représentent ces objets en tendant à être au plus près du réel alors que celles stylisées illustrent ces objets du réel de façon plus ou moins modifiée. Les formes d'art abstrait, quant à elles, ne représentent pas des objets du réel. Ses représentations sont non-objectives. Ainsi, seul l'imaginaire du spectateur peut lui attribuer une représentation réaliste. Concernant le jardin en lui-même, sa superficie est divisée en quatre carrés illustrant des invariants culturels : la Terre, l'Eau, le Feu et le Vent. Les composants de l'aménagement, retrouvés dans chacun de ces carrés, symbolisent la thématique de l'invariant culturel par les couleurs des végétaux et par les œuvres qui font aussi référence à la culture régionale.

Le travail que nous allons présenter ici, à partir de l'observation de six cas cliniques, a pour objectif principal de révéler en profondeur l'expérience vécue par les patients en déterminant les effets de la dimension artistique et végétale de ce jardin sur leurs processus mentaux et émotionnels. 
Figure 1. Illustration non-exhaustive d'œuvres du jardin "art, mémoire et vie" (titre de l'œuvre, forme d'art, représentations, matériaux)
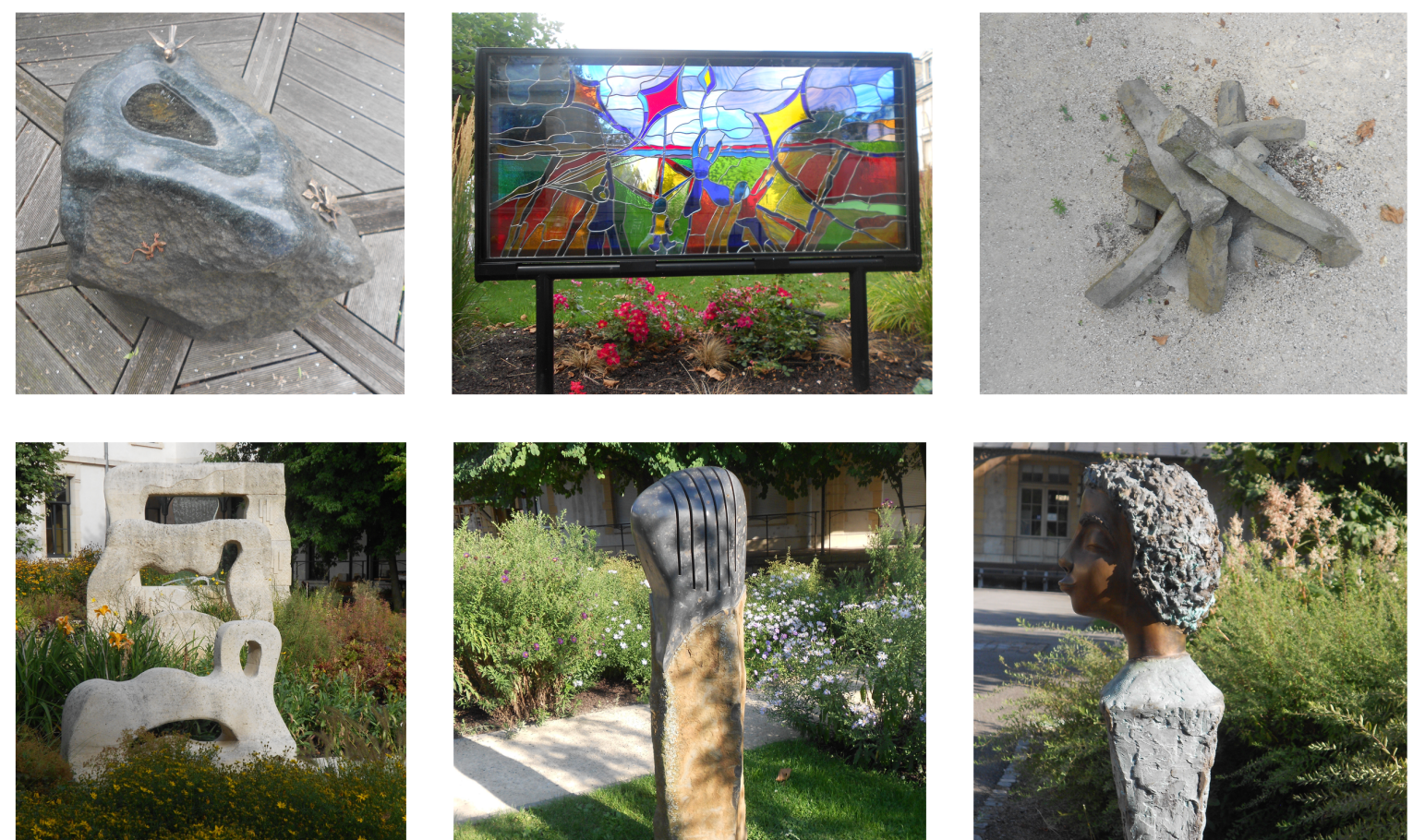

Légende: De gauche à droite: En haut. L'Abreuvoir, figuratif, représentations mimétiques, olivine de Hesse, basalte et éléments en bronze (i.e. oiseau, lézard, papillon) ; Vitrail, figuratif, représentations fortement stylisées, verre plombé ; Feu de camp, figuratif, représentations mimétiques, tuf de Hesse. En bas. Cycle, abstrait, représentations non-objectives, pierre de Lérouville ; Klangstein, abstrait, représentations non-objectives, grès et basalte ; Le Bisou, figuratif, représentations stylisées, bronze.

\section{Hypothèses}

Nous émettons l'hypothèse générale selon laquelle la perception des œuvres d'art et des végétaux implantés dans ce jardin sollicite les processus mnésiques (mémoire épisodique et sémantique) des patients MAMA et favorise l'expression d'émotions positives (joie et surprise) au cours d'une promenade accompagnée.

Hypothèses opérationnelles

- Si l'on analyse le support végétal versus artistique séparément alors :

Il existe un lien entre le type d'élément perçu, végétal versus œuvres d'art, et :

○ La forme de mémoire mobilisée, sémantique versus épisodique (H1).

○ La qualité émotionnelle suscitée, joie versus surprise $(\mathrm{H} 2)$.

Devant une œuvre d'art (H3) ou devant un végétal (H4) :

- la mémoire sémantique est plus mobilisée que la mémoire épisodique.

- l'émotion de joie est plus souvent exprimée que l'émotion de surprise.

- Si l'on compare le type d'élément perçu, végétal versus œuvre d'art, devant cette dernière :

○ la mémoire sémantique (H5) ou épisodique (H6) est plus mobilisée.

○ l'émotion de joie ou de surprise (H7) est plus souvent exprimée. 
Le mode d'installation des troubles de la MA étant marqué au premier plan par une altération de la mémoire épisodique [1], nous supposons que cette mémoire sera par conséquent moins mobilisée que la mémoire sémantique quelque soit le support perçu. Sur le plan émotionnel, nous pensons que les éléments perçus, artistiques ou végétaux, vont majoritairement provoquer une émotion de joie répondant à un des objectifs du jardin ayant guidé son aménagement : favoriser le bien-être des usagers [16, 17].

\section{Méthode}

\section{Population}

Notre population est constituée de six patients (tableau 1) : trois hommes et trois femmes atteints de MAMA aux stades modéré à sévère correspondants aux critères d'éligibilité (tableau 2). Pour ces six patients, un diagnostic de MA a été porté et pour trois d'entre eux, une composante vasculaire (démence mixte) s'y associe. Les sujets sont âgés en moyenne de 81 ans (extrêmes : 72-90) et vivaient à domicile en Lorraine avant leur hospitalisation. Chaque sujet a été vu par une psychologue une seule fois dans le cadre de son hospitalisation au Centre Paul Spillmann du CHRU de Nancy. Ils ont par ailleurs bénéficié d'une évaluation neuropsychologique standard (tableau 1) pour déterminer leur profil cognitif.

Cette recherche exploratoire a été conduite après avoir reçu l'avis favorable du Comité de Réflexion Ethique Nancéien Hospitalo-Universitaire (CRENHU).

Tableau 1 - Caractéristiques socio-démographiques et données médicales et neuropsychologiques des patients

\begin{tabular}{|c|c|c|c|c|c|c|}
\hline & Mme D & Mme P & Mme V & $\mathrm{MrB}$ & Mr D & $\mathrm{Mr} \mathrm{G}$ \\
\hline$\hat{A g e}$ & 80 & 90 & 72 & 83 & 80 & 83 \\
\hline Niveau socio-culturel & 2 & 2 & 3 & 3 & 2 & 2 \\
\hline Statut matrimonial & $\mathrm{V}$ & $\mathrm{V}$ & $\mathrm{V}$ & $\mathrm{V}$ & M & M \\
\hline Diagnostic & Mixte & MA & Mixte & MA & MA & Mixte \\
\hline Stade de démence & Modéré & Modéré & Modéré & Modéré & Sévère & Modéré \\
\hline Score au MMSE et à ses subtests & $14 / 30$ & $19 / 30$ & $16 / 30$ & $12 / 30$ & $7 / 30$ & $17 / 30$ \\
\hline Orientation tempo-spatiale & $3 / 10$ & $5 / 10$ & $3 / 10$ & $2 / 10$ & $1 / 10$ & $3 / 10$ \\
\hline Apprentissage & $3 / 3$ & $3 / 3$ & $3 / 3$ & $2 / 3$ & $2 / 3$ & $3 / 3$ \\
\hline Attention / Calcul & $0 / 5$ & $3 / 5$ & $1 / 5$ & $0 / 5$ & $0 / 5$ & $0 / 5$ \\
\hline Rappel & $0 / 3$ & $0 / 3$ & $0 / 3$ & $1 / 3$ & $0 / 3$ & $3 / 3$ \\
\hline Langage & $8 / 8$ & $8 / 8$ & $8 / 8$ & $7 / 8$ & $4 / 8$ & $7 / 8$ \\
\hline Visuo-construction & $0 / 1$ & $0 / 1$ & $1 / 1$ & $0 / 1$ & $0 / 1$ & $1 / 1$ \\
\hline \multicolumn{7}{|l|}{ Mémoire épisodique verbale } \\
\hline$\overline{\text { Score global Epreuve des } 5 \text { mots }}$ & $7 / 10$ & $5 / 10$ & $3 / 10$ & $\mathrm{DM}$ & $1 / 10$ & $8 / 10$ \\
\hline Score d'apprentissage & $4 / 5(3+1)$ & $3 / 5(2+1)$ & $3 / 5(3+0)$ & $\mathrm{DM}$ & $1 / 5(0+1)$ & $5 / 5(3+2)$ \\
\hline Score de mémoire & $3 / 5(0+3)$ & $2 / 5(2+0)$ & $0 / 5(0+0)$ & $\mathrm{DM}$ & $0 / 5$ & $3 / 5(3+0)$ \\
\hline Intrusion & 1 & 0 & 0 & DM & 2 & 0 \\
\hline Score à la BREF et à ses subtests & $6 / 18$ & $7 / 18$ & $9 / 18$ & DM & $8 / 18$ & $6 / 18$ \\
\hline$\overline{\text { Similitudes }}$ & $0 / 3$ & $0 / 3$ & $1 / 3$ & DM & $0 / 3$ & $1 / 3$ \\
\hline Fluences verbales & $0 / 3$ & $1 / 3$ & $0 / 3$ & DM & $0 / 3$ & $0 / 3$ \\
\hline Comportement de préhension & $0 / 3$ & $3 / 3$ & $3 / 3$ & DM & $3 / 3$ & $0 / 3$ \\
\hline Séquences motrices de Luria & $3 / 3$ & $1 / 3$ & $1 / 3$ & DM & $1 / 3$ & $3 / 3$ \\
\hline Consignes conflictuelles & $1 / 3$ & $2 / 3$ & $3 / 3$ & DM & $2 / 3$ & $1 / 3$ \\
\hline Go-NoGo & $2 / 3$ & $0 / 3$ & $1 / 3$ & DM & $2 / 3$ & $1 / 3$ \\
\hline
\end{tabular}




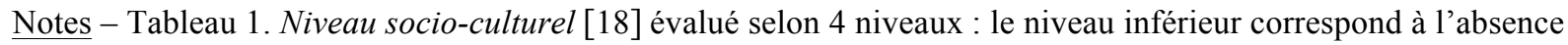
de diplôme voire à l'obtention d'un CAP et le niveau le plus élevé représente l'obtention au minimum du BAC. Statut matrimonial : veuf(ve) - vit seul(e) (V); marié(e) - vit en couple (M). Diagnostic : Démence mixte : MA à composante vasculaire (Mixte) ; Démence de type Alzheimer (MA).

Score au MMSE : Mini mental state examination (MMSE) de Folstein [19]; Mémoire épisodique verbale: Epreuve des 5 Mots de Dubois [20]; Score à la BREF : Batterie rapide d'évaluation frontale [21]; Données manquantes (DM).

Tableau 2 - Critères d'éligibilité

\begin{tabular}{|c|c|}
\hline Critères d'inclusion & Critères de non-inclusion \\
\hline $\begin{array}{l}\text { - âgés entre } 65 \text { et } 95 \text { ans } \\
\text { - marchent de manière autonome, c'est-à-dire sans aide, } \\
\text { qu'elle soit humaine ou technique, hormis une simple canne } \\
\text { - hospitalisés au Centre Paul Spillmann } \\
\text { - avoir déjà fréquenté le jardin seul et/ou accompagné au } \\
\text { moins } 15 \text { jours avant l'entretien } \\
\text { - ont donné leur consentement écrit après avoir reçu une } \\
\text { information orale et écrite, claire et intelligible } \\
\text { - diagnostic de MA probable à un stade modéré à sévère } \\
\text { d'après les critères diagnostiques du DSM-IV [2] et du } \\
\text { NINCDS-ADRDA [22] } \\
\text { - score au MMSE [19] compris entre } 7 \text { et } 20 \text { dans les } 2 \text { mois } \\
\text { précédant l'inclusion } \\
\text { - traitement médicamenteux spécifique de la MA, stable } \\
\text { depuis au moins } 2 \text { mois (anticholinestérasique ou } \\
\text { mémantine) } \\
\text { - absence de modification du traitement psychotrope dans } \\
\text { les } 48 \text { hrécédant l'inclusion }\end{array}$ & $\begin{array}{l}\text { - déficit sensoriel et/ou troubles phasiques interférant avec la } \\
\text { tâche envisagée dans l'étude } \\
\text { - troubles comportementaux sévères } \\
\text { - refus ou impossibilité de recueil écrit du consentement } \\
\text { éclairé du sujet } \\
\text { - autres maladies neurologiques ou psychiatriques pouvant } \\
\text { modifier l'évaluation } \\
\text { - dépression sévère }\end{array}$ \\
\hline
\end{tabular}

\section{Situation de communication et contexte de l'entretien}

Chaque participant était invité à se promener avec la psychologue dans le jardin. Son rythme était respecté, il choisissait son parcours ainsi que les éléments devant lesquels il souhaitait s'arrêter. Tous les échanges étaient audio-enregistrés afin de capter tous les commentaires des patients, qu'ils soient produits librement ou sollicités par les relances de la psychologue.

Dans cette étude exploratoire nous conjuguons ainsi trois principes : i) dialoguer ii) dans un jardin iii) intégrant une dimension artistique.

\section{Analyse}

Notre démarche repose sur l'analyse interlocutoire [15] du discours des sujets afin de mettre en évidence les processus mnésiques (mémoire épisodique ou sémantique) ou émotionnels (surprise ou joie) suscités par le support perçu (végétal ou œuvre d'art) (figure 2). L'unité discursive est l'énoncé, véritable trace émotionnelle et de l'évocation des connaissances générales et des souvenirs personnels. Une analyse statistique a ensuite été réalisée afin de tester nos hypothèses (Khi-deux de Pearson pour tester le lien entre les variables et $t$-test pour les comparaisons de moyennes pour échantillons appariés (logiciel SPSS 12)). 
Figure 2. Information psychologique transportée dans l'énonciation du patient selon qu'il se trouve devant un végétal ou devant une œuvre d'art

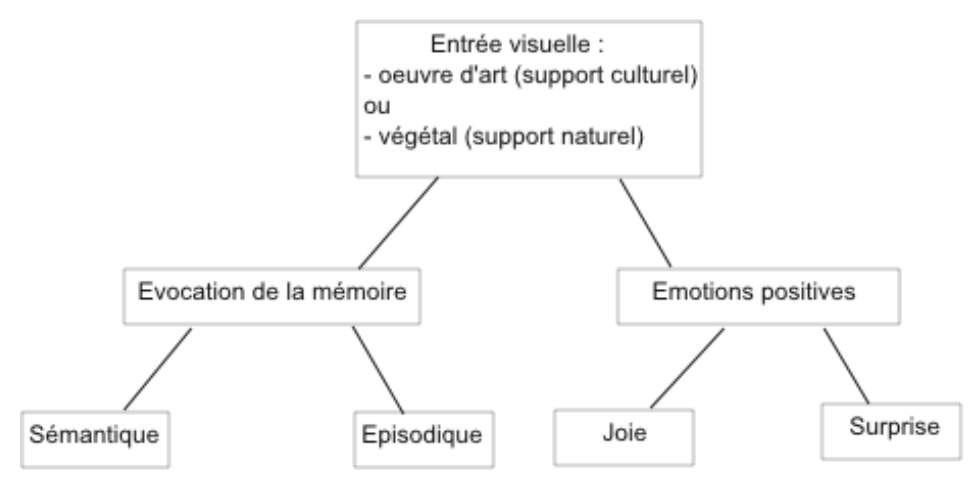

\section{Résultats globaux}

La durée moyenne d'une promenade est de 48,82 mn (extrêmes : 34,01-68,24).

\section{Résultats qualitatifs}

Pour répondre à notre hypothèse générale, nous présentons les résultats qualitatifs, illustrant l'ensemble des processus mnésiques (mémoire sémantique, épisodique, collective) et émotionnels (émotions positives de joie et de surprise) en lien avec la perception de la dimension végétale et artistique du jardin.

\subsection{Compétences mnésiques}

\section{Mémoire sémantique}

La vue d'un élément du jardin, végétal ou artistique, peut activer la récupération de connaissances générales qui n'ont pas été antérieurement énoncées par la psychologue.

- Mémoire sémantique et perception des végétaux : Pour tous les patients, la perception de végétaux suscite la dénomination de leur catégorie d'appartenance formalisée par des hyperonymes (e.g., «arbre $(s) », \quad$ "plante $(s) », \quad$ fleur $(s) »)$ et des hyponymes sémantiquement vrais. Certains de ces hyponymes peuvent être bien (re)connus même par des profanes en horticulture ou botanique (e.g. "roses", "cerisier», "lavande») et d'autres dénotent une connaissance approfondie dans ce domaine en particulier chez deux patients (e.g, «althéa », "saxifrages»). L'expression de stimulations sensorielles (vue, odeur) est manifestée (e.g., «oh l'parfum!») outre des descriptions particulièrement précises. Certains patients vont même partager des conseils concernant l'entretien des végétaux et évoquer leur utilité éventuelle.

- Mémoire sémantique et perception des œuvres d'art: Devant des œuvres, les patients s'expriment sur: leurs matériaux composites (e.g. "minerai», "roche», "verre », «granit») ou leur provenance, leurs modes de conception ou décrivent ce qu'elles 
représentent. A titre d'exemple, devant L'Abreuvoir certains sujets vont dénommer correctement ses éléments en bronze qui représentent de façon mimétique des animaux (figure 1). La perception d'œuvres figuratives stylisées ou abstraites conduit les sujets à dénommer aussi les formes qui y sont représentées ou partagent ce qu'ils imaginent (e.g., « des enfants », « une nymphe », « une girafe », " p'lotes basques », " une balance »).

\section{Mémoire épisodique}

$\mathrm{Au}$ cours de la promenade, les patients s'expriment sur leur fréquentation du jardin, leur parcours habituel, les endroits visités, ou non, ainsi que des éléments du jardin qu'ils ont éventuellement déjà perçus. Se promener dans ce jardin stimule ainsi des réminiscences des souvenirs individuels. Cette situation va offrir aussi aux patients l'opportunité de partager des informations socio-démographiques ou de transmettre le rapport qu'ils entretiennent avec le jardin en général, le leur, ou les activités qu'ils y pratiquent habituellement. Outre le partage de ces informations associées à l'usage de cet environnement, des patients se remémorent des souvenirs personnels qui sont en lien direct avec la perception de certains végétaux lors de la promenade.

- Mémoire épisodique et perception des végétaux: Quatre sujets partagent avec l'interviewer leurs souvenirs de certains végétaux perçus (e.g., « un arbre à papillons on en on en a plusieurs (...) on en a même un beau blanc »). D'autres évoquent des expériences vécues comme par exemple, la vue des roses qui active un souvenir qui lui est liée (e.g., " $p^{\prime} i s y^{\prime}$ a des roses à gogo des belles roses. Alors y'en a des belles euh oranges là. Alors je dis à mon fils "oh la la les belles roses!»).

- Mémoire épisodique et perception des œuvres d'art : La dimension artistique, tout comme la dimension végétale, a un potentiel d'évocation mnésique d'expériences individuelles. Trois patients expriment un sentiment de familiarité ou de « déjà vu» (e.g. " j'ai déjà vu chez des gens ", "j'l'avais aperçu mais j'étais pas v'nu encore », "oui j'ai vu. (...) Y'a longtemps qu'j'ai $v u »)$.

\section{Mémoire collective}

La mémoire collective est stimulée lors de cette tâche chez deux patients devant des œuvres. Un patient évoque des histoires de la seconde Guerre mondiale ou des festivités culturelles régionales. Les minéraux des sculptures du jardin vont éveiller la mémoire collective du patrimoine géologique régional chez ces deux sujets (e.g., «pierres qui viennent de soit des Vosges», "cailloux d'la Moselle»). Alors qu'il voit un banc qu'il qualifie de «banc d'amoureux », le patient va chantonner « sur les bancs de la voie publique voie publique » sur l'air de la chanson 'Les amoureux des bancs publics"' de Brassens. L'autre patient va évoquer Picasso en parlant des sculptures perçues.

\section{2. Processus émotionnels}

\section{Emotions positives}

La promenade dans le jardin semble favoriser l'expression verbale d'émotions positives : la joie et la surprise. 


\section{Surprise}

Contrairement à la joie qui est toujours attribuée à une émotion positive, la surprise peut être positive ou négative. L'émotion de surprise a été catégorisée positive lorsqu'elle précédait ou suivait l'expression de réflexions, de descriptions ou de jugements de valeur neutres ou positifs à propos d'un élément perçu.

- Devant des œuvres d'art : Nous avons répertorié chez cinq patients la surprise suscitée par la perception d'éléments artistiques. C'est sur le registre affectif que deux patients s'expriment devant quatre œuvres figuratives stylisées et une abstraite (i.e. "c'est étonnant», "ça fait drôle », "pierre qui est drôl'ment drôle », «drôles de pierres »). La surprise est verbalisée par des émotimots [23] chez trois patients qui sont des indicateurs d'une émotion de surprise ressentie (e. g., «oh la la! », «ouh la la! ben dis donc! », «ah bon? », «ah?»). Ces interjections précèdent des descriptions ou des jugements. D'autres font suite aux explications ou aux expressifs d'accord formulés par la psychologue.

- Devant des végétaux: Nous faisons le même constat chez trois patients devant des végétaux (e.g., "ah?», «ah bon. Ah!», "Oh mon Dieu. Qu'est-c'que c'est $q u ' c ̧ a ?$ (silence) ah on dirait des des (silence) regardez ça.»).

\section{Joie}

- Devant des œuvres d'art : Tous les sujets expriment de la joie devant les stimuli artistiques: des rires, des expressions verbales, des chantonnements ou des fredonnements de mélodies. Quatre des six sujets vont exprimer cette émotion (i.e. " $i l$ est comme moi il est heureux", "c'est mon plus grand bonheur de regarder les oiseaux », " ben j'trouve ça c'est rigolo » "Non. Je rigole. " «c'est marrant hein? »). Certains rires sont partagés dans le dialogue après des réminiscences ou après des explications de la psychologue. On relève également des plaisanteries comme, par exemple, chez une patiente : «j'la vol'rai avant d'partir», «j'vais l'prendre» en précisant ensuite qu'elle « rigole».

- Devant des végétaux : Après avoir vu un végétal, quatre sujets expriment de la joie par des rires et/ou des mélodies. Les sujets rient personnellement ou parfois après des questions de la psychologue qui visent à solliciter le patient à dire ce qu'il perçoit dans le jardin. Des actions, qu'on peut retrouver dans des situations courantes menées dans un jardin, comme d'écarter les fleurs pour se frayer un passage ou de donner un brin de lavande, sont présentes lors de cette promenade. Ces actions sont suivies de rires dans les deux entretiens qui sont concernés. C'est d'ailleurs après avoir donné un brin de lavande que le patient va chantonner « souvenirs souvenirs » en riant.

\section{Emotions négatives}

Des émotions négatives, telles que la peur ou de la tristesse, ont également été retrouvées chez certains sujets. Elles ne concernent ni la dimension artistique, ni les végétaux perçus. La tristesse est générée par la perception visuelle de l'extérieur (ville). Cette perception de la ville va engendrer ensuite des réminiscences positives (remémoration d'un souvenir agréable) et la production de données autobiographiques. La peur est retrouvée dans trois entretiens : la 
peur de se perdre dans le jardin, de tomber en marchant ou en s'asseyant sur un banc et la peur que l'hélicoptère en vol lui tombe dessus.

\section{3. Autres processus psychologiques}

D'autres processus psychologiques sont retrouvés au sein de ces entretiens lorsque les patients contemplent des œuvres ou des végétaux du jardin : l'imagination, des réflexions introspectives et un phénomène d'imitation.

\section{Imagination}

L'imagination, en lien avec la mémoire [24], peut avoir pour support matériel l'œuvre d'art dans la construction de scénarii. Face à une œuvre figurative, l'expression des représentations chez tous les sujets est stimulée mais aussi :

- des états émotionnels. Par exemple, la perception visuelle de l'oiseau en bronze sur L'Abreuvoir (figure 1), en plus des stimulations mnésiques, va solliciter l'imaginaire d'une patiente sur le plan émotionnel (i.e. « il est comme moi il est heureux »)

- des actions ou des éléments kinesthésiques produits par des éléments représentant du vivant (e.g., "il r'monte », « il va boire un coup »)

- des intentions attribuées aux éléments figuratifs qui, par conséquent, sont personnifiés (e.g., « il veut aller dans la piscine », «il veut aller becter»)

- des attributs vestimentaires, des caractéristiques physiques ou physiologiques précises attribuées là-encore aux objets d'art, phénomène projectif par excellence (e.g., « $y^{\prime} a$ une femme », « enceinte », " il est costaud»).

Dans tous les entretiens, les œuvres abstraites ou les bancs vont éveiller l'imagination lorsque les patients leurs attribuent des représentations figées (e.g., «une goutte d'eau», «des doigts », « un peigne », « des boucles d'oreilles », « elle ressemble à moi », " bouche », " tête d'un sanglier », "une corbeille») ou kinesthésiques (e.g., "on croirait une main qui s'tient qui tient le le le le pavé là.»). Certains patients, vont imaginer ou projeter :

- l'usage qu'eux-mêmes ou autrui pourrait se faire d'une œuvre s'ils en avaient possession ou s'ils l'approchaient (e.g., " ça avec une boîte d'allumettes à chaque gosse (silence) à un vieux paquet pour qu'ils puissent faire du feu (...) ils s'raient heureux mais p't'être pas tout l'monde (en riant)», "oh pour mettre pour mettre des lapins (...) des lapins en d'ssous p'is au d'sus le foin »)

- des projections sur le concepteur du jardin ou son savoir-faire (e.g., " celui qui a fait ça et ben il fait un il rêvait », "c'ui qui l'a fait y'a fallu qu'il le r'mue et puis (silence) qu'il le façonne »)

- la provenance des matériaux composites.

La perception de certains végétaux du jardin va, elle aussi, être source d'imagination chez quatre patients qui abordent l'évolution des fleurs (e.g., " elle va grimper ») ou leurs couleurs quand les bourgeons auront éclos (e.g., «vous voyez tous ceux qui sont verts là-bas ? (...) eh ben $i^{\prime}$ vont d'v'nir bleus. »). En imaginant ces situations, ils évoquent le sentiment esthétique que cela produirait («ça ça va être beau quand ça va fleurir »). 


\section{Réflexions introspectives et (auto-)questionnements}

Devant les œuvres du jardin, tous les sujets vont manifester des réflexions introspectives (tableau 3) sur :

- leurs fonctions intrinsèques et leur fonctionnement,

- leurs représentations,

- la nature de leurs matériaux et leur provenance d'origine

- ou le statut du créateur.

La végétation suscite des réflexions menées par tous les sujets qui se révèlent dans le dialogue. Les sujets posent des questions à propos de leur appartenance catégorielle ou sur la saison et le jardinage (e.g., "comment ça s'appelle? », "qu'est-c'que c'est? ", "c'est pas *nom du végétal*?», «c'est pas maint'nant l'moment des r'piquages?»).

Tableau 3. Illustrations des réflexions introspectives et des (auto-)questionnements à propos des œuvres d'art

\begin{tabular}{|c|c|}
\hline Réflexions introspectives sur & Illustrations \\
\hline - Leurs fonctions intrinsèques & 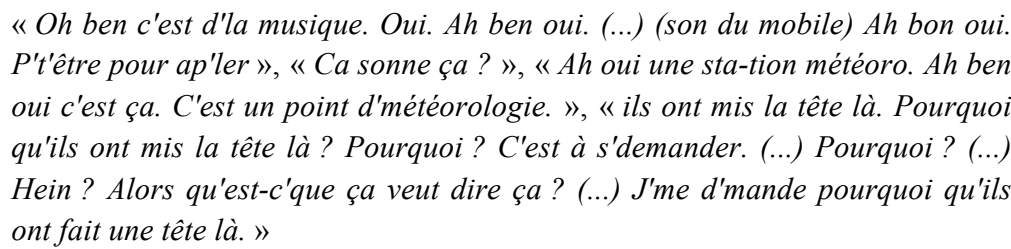 \\
\hline - Leur fonctionnement & $\begin{array}{l}\text { «En cas de grosses gelées alors là c'est (silence) Ch'ais pas comment ça } \\
\text { marche.», «Comment elle repart et puis elle revient? C'est ça qui faut voir. » }\end{array}$ \\
\hline - Leurs représentations & $\begin{array}{l}\text { "Oh c'est quoi un bac à sable ça?», «Ca r'présente quelque chose mais } \\
\text { quoi ?», «Ca r'présente quoi? Qu'est-c'qu'ça peut r'présenter? », « On dirait } \\
\text { on dirait mais c'est pas une femme ça. Ah oui.», «Alors ça (silence) j'sais pas } \\
\text { c'que ça r'présente ça s'rait pas une forteresse ça? ", «ben ça (touche) } \\
\text { (silence) ça ce s'rait la la sainte vierge non?» }\end{array}$ \\
\hline $\begin{array}{l}\text { - La nature de leurs matériaux et leur } \\
\text { provenance d'origine }\end{array}$ & $\begin{array}{l}\text { "C'est d'la planche? ", «Ca c'est une euh petite une ferraille pas une ferraille } \\
\text { euh un rocher qui ont r'trouvé en profondeur.», «Ca doit être plombé hein?» }\end{array}$ \\
\hline - Le statut du créateur & «tout tout p'is ça ça doit être un artiste hein?» \\
\hline
\end{tabular}

\section{Imitation}

La sculpture Le Bisou (figure 1) invite deux patients à reproduire par une mimique ou par des bruits le comportement qui y est associé. En dehors de ces deux sujets, aucun autre comportement d'imitation ne s'est produit. Nous pouvons ainsi émettre l'hypothèse selon laquelle la composante affective forte générée par l'œuvre est à l'origine de ce phénomène d'imitation.

L'ensemble de ces résultats qualitatifs confirme notre hypothèse générale. Nous présentons maintenant des extraits de l'analyse du cas de $\mathrm{Mr} \mathrm{G}$ qui illustrent combien ces principaux processus cognitifs et émotionnels sont intégrés dans le dialogue qui est suscité par le jardin. 


\section{Illustration par un cas clinique : Mr G}

Mr G, âgé de 83 ans, est hospitalisé pour une poursuite de la prise en charge d'une suspicion d'accident vasculaire cérébral avec broncho-pneumopathie. Il souffre d'une démence mixte suivie et traitée. Son évaluation neuropsychologique (tableau 1) met en évidence une altération de l'efficience cognitive globale à un stade modéré avec un MMSE à 17/30 [19].

Premier exemple concernant M. G : La perception d'un végétal va solliciter à la fois la mémoire sémantique et épisodique au sein d'un seul tour de parole. Lorsque son regard se dirige vers le jasmin d'hiver, Mr G informe la psychologue qu'il en possède un puis précise le nom de la plante perçue.

Deuxième exemple concernant M. G (Encadré 1) : La perception des végétaux, au-delà des connaissances sémantiques, suscite la mémoire épisodique de ce patient et génère des émotions positives. A titre d'exemple, devant un buddleia, il indique qu'il ne connaît pas le «nom scientifique» de la plante (par un mouvement d'introspection épistémique) mais évoque, en riant (exprimant ainsi une émotion positive), des souvenirs personnels (expression de la mémoire épisodique) qui lui sont liés.

Encadré 1 - Intégration multimodale de la mémoire épisodique et sémantique accompagnée d'émotions de joie

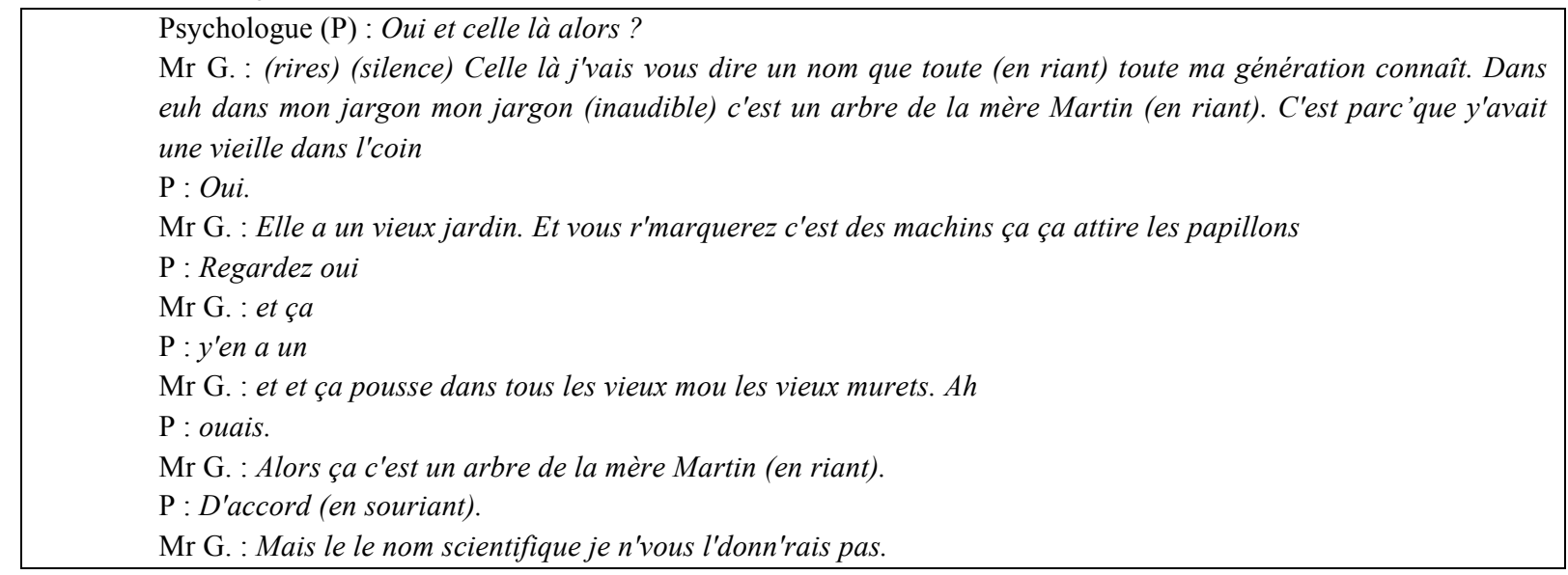

Exemple 3. (Encadré 2, Figure 3) : Dans cet exemple, là encore, la vue des œuvres d'art est propice à l'émergence de processus mentaux dans une activité intégrative par l'activation multimodale de la mémoire (sémantique, épisodique et des événements socio-culturels) associée à la verbalisation de jugement esthétique, du recours à l'imaginaire et de réflexions. 
Encadré 2. Activité intégrative par l'activation multimodale de la mémoire (sémantique, épisodique et des événements socio-culturels) associée à la verbalisation de jugement esthétique, du recours à l'imaginaire et de réflexions

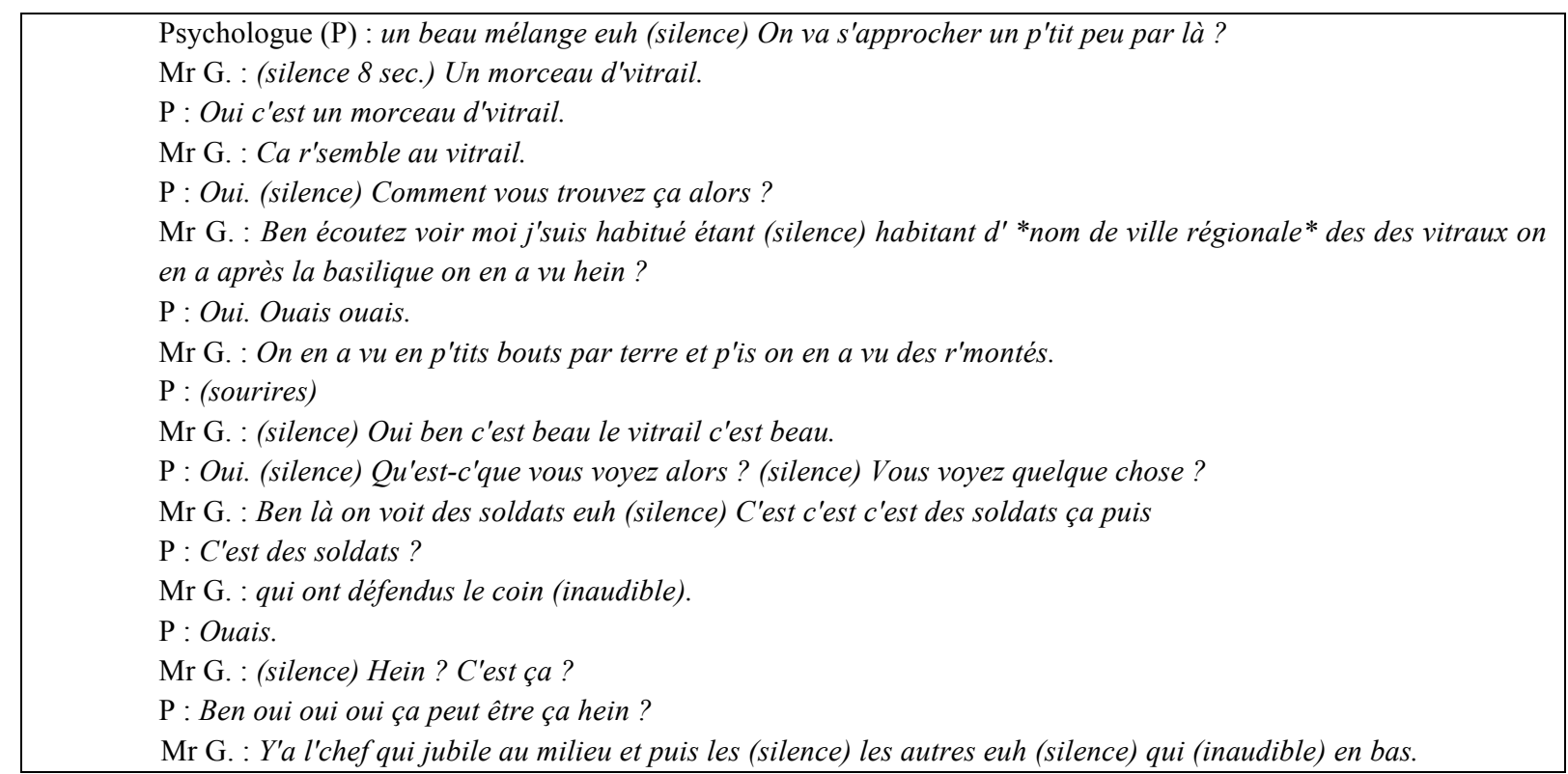

Figure 3. Activité multimodale des processus mentaux depuis la perception du Vitrail

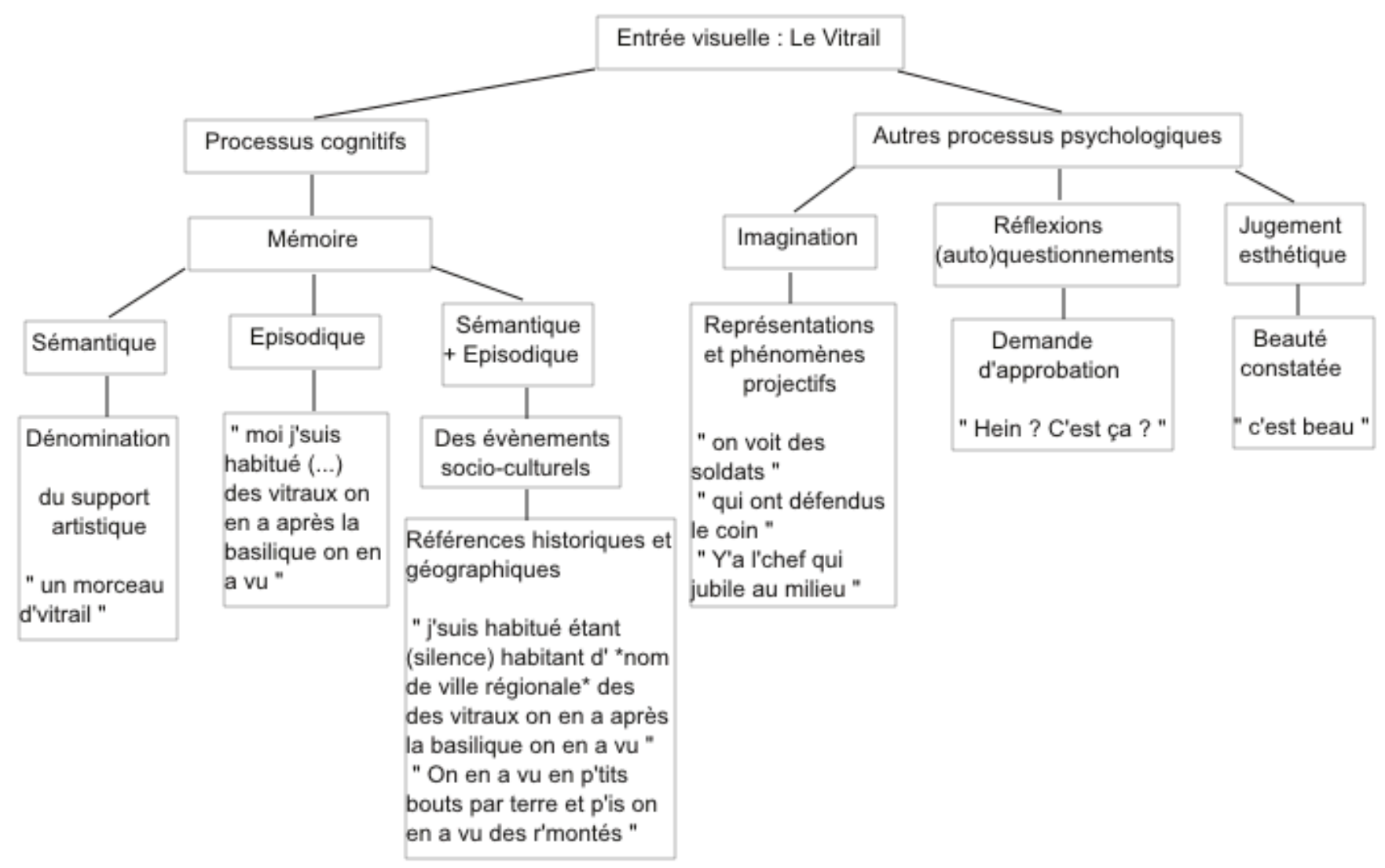




\section{Résultats quantitatifs}

Nous avons analysé 1258 énoncés issus des 6 entretiens.

- Lorsque l'on analyse le support végétal versus artistique séparément :

H1 : Il existe un lien statistiquement significatif entre le type d'élément perçu, végétal versus artistique, et la forme de mémoire mobilisée, sémantique versus épisodique (Khi-deux de Pearson $=14,32 ; \mathrm{p}<0,001 ; \mathrm{Phi}=0,12)($ tableau 4$)$.

Tableau 4. Occurrences d'énoncés émis relevant des différentes formes de mémoire sollicitées et des différents types d'émotions suscités en fonction du type de support perçu.

\begin{tabular}{|c|c|c|c|c|c|c|c|}
\hline \multirow{5}{*}{ Support } & & \multicolumn{2}{|c|}{$\begin{array}{l}\text { Mémoire } \\
\text { sollicitée }\end{array}$} & \multicolumn{2}{|c|}{$\begin{array}{c}\text { Emotion } \\
\text { suscitée }\end{array}$} & \multirow{2}{*}{$\begin{array}{l}\boldsymbol{p} \\
\text { Support } v s \\
\text { Mémoires }\end{array}$} & \multirow[b]{2}{*}{$\begin{array}{l}\text { Support } v s \\
\text { émotions }\end{array}$} \\
\hline & & ME & MS & JOIE & SURPRISE & & \\
\hline & Art & $\begin{array}{c}109 \\
(19 \%)\end{array}$ & $\begin{array}{c}466 \\
(81 \%)\end{array}$ & $\begin{array}{c}123 \\
(71 \%)\end{array}$ & $\begin{array}{c}50 \\
(29 \%)\end{array}$ & $<0,001$ & $\mathrm{p}=0,054$ \\
\hline & Végétal & $\begin{array}{c}137 \\
(29 \%)\end{array}$ & $\begin{array}{c}337 \\
(71 \%)\end{array}$ & $\begin{array}{c}20 \\
(56 \%)\end{array}$ & $\begin{array}{c}16 \\
(44 \%)\end{array}$ & & \\
\hline & Total & 246 & 803 & 143 & 66 & & \\
\hline
\end{tabular}

Notes: ME : énoncés relevant de la mémoire épisodique ; MS : énoncés relevant de la mémoire sémantique ; JOIE : énoncés relevant de la manifestation émotionnelle de joie; SURPRISE : relevant de la manifestation émotionnelle de surprise; Art : œuvre d'art perçue (support artistique) ; Végétal : végétal perçu (support végétal); En gras : résultat statistiquement significatif.

$\mathrm{H} 2$ : On note une tendance à la significativité entre le type d'élément perçu, végétal versus artistique, et la qualité émotionnelle suscitée, joie versus surprise (Khi-deux de Pearson = $3,33 ; \mathrm{p}=0,054 ; \mathrm{Phi}=0,126)$. Si les occurrences d'énoncés à valeur émotionnelle étaient plus importantes, il aurait été probable de constater l'existence d'un lien significatif (tableau 4).

H3 : Devant une œuvre d'art, la mémoire sémantique est plus mobilisée que la mémoire épisodique $(t[1,5]=3,526, \mathrm{p}=0,0085)$ et l'émotion suscitée relève plus de la joie que de la surprise au risque $3,4 \%$ (tableau 5).

H4 : Devant un végétal, on ne pas conclure à une différence significative entre le type de mémoire sollicitée, sémantique versus épisodique. On note cependant une tendance à la significativité $(5,3 \%)$ dans le sens d'une mémoire sémantique plus mobilisée que la mémoire épisodique. Sur le plan émotionnel, on ne peut pas conclure à une différence significative concernant le type d'émotion suscitée. La qualité de l'émotion suscitée par le végétal relève autant de la joie que de la surprise (tableau 5). 
Tableau 5 - moyenne d'énoncés émis relevant des différentes formes de mémoire sollicitées et des différents types d'émotions suscités en fonction du type de support perçu.

\begin{tabular}{lccccll}
\hline & ME & MS & JOIE & SURPRISE & p & \\
& M $+\mathrm{DS}$ & $\mathrm{M}+\mathrm{DS}$ & $\mathrm{M}+\mathrm{DS}$ & $\mathrm{M} \pm \underline{\mathrm{DS}}$ & $\mathrm{MS}$ & JOIE $v s$ \\
& & & & & SURPRISE \\
Art & $18,2 \pm 11$ & $77,7 \pm 39,5$ & $20,5 \pm 14,2$ & $8,3 \pm 6,6$ & $\mathbf{0 , 0 0 8 5}$ & $\mathbf{0 , 0 3 4}$ \\
Végétal & $22,8 \pm 22,1$ & $56,2 \pm 45$ & $3,3 \pm 3,9$ & $2,7 \pm 2,5$ & 0,053 & 0,393 \\
& & & & & \\
\hline
\end{tabular}

Notes - Tableau 5. ME : moyenne d'énoncés relevant de la mémoire épisodique ; MS : moyenne d'énoncés relevant de la mémoire sémantique; JOIE : moyenne d'énoncés relevant de la manifestation émotionnelle de joie ; SURPRISE : moyenne d'énoncés relevant de la manifestation émotionnelle de surprise ; Art : œuvre d'art perçue (support artistique) ; Végétal : végétal perçu (support végétal) ; $\mathrm{M}$ : moyenne ; DS : déviation standard ; En gras : résultat statistiquement significatif.

- Comparaison de l'impact du type de support perçu (œuvre d'art versus végétal) :

H5 : On peut affirmer au risque $1,2 \%$ que devant une œuvre, la mémoire sémantique est plus mobilisée que devant un végétal $(t[1,5]=3,189, \mathrm{p}=0,012)$ (tableau 6).

H6: On ne peut pas conclure à un impact du type de support sur la mobilisation de la mémoire épisodique. La mémoire épisodique ne semble pas plus mobilisée devant une œuvre d'art qu'un végétal (tableau 6).

H7 : On peut affirmer, au risque $0,6 \%$, que la joie est plus souvent exprimée devant une œuvre d'art que devant un végétal $(t[1,5]=3,888, \mathrm{p}=0,006)$, tout comme la surprise $(t[1,5]=$ 2,077, $\mathrm{p}=0,046$ ) (tableau 6).

Tableau 6. Impact du support présenté sur le nombre moyen d'énoncés émis relevant de chaque type de mémoire et de chacune des émotions.

\begin{tabular}{lccc}
\hline & $\begin{array}{c}\text { Art } \\
(\mathrm{M} \pm \mathrm{DS})\end{array}$ & $\begin{array}{c}\text { Végétal } \\
(\mathrm{M}+\mathrm{DS})\end{array}$ & $\begin{array}{c}\mathbf{p} \\
\text { Art } v s \text { Végétal }\end{array}$ \\
ME & $18,2 \pm 11$ & $22,8 \pm 22,1$ & 0,223 \\
MS & $77,7 \pm 39,5$ & $56,17 \pm 45$ & $\mathbf{0 , 0 1 2}$ \\
JOIE & $20,5 \pm 14,2$ & $3,3 \pm 3,9$ & $\mathbf{0 , 0 0 6}$ \\
SURPRISE & $8,3 \pm 6,6$ & $2,7 \pm 2,5$ & $\mathbf{0 , 0 4 6}$ \\
\hline
\end{tabular}

Notes : ME : mémoire épisodique ; MS : mémoire sémantique ; JOIE : Joie ; SURPRISE : surprise ; Art : œuvre d'art perçue (support artistique) ; Végétal : végétal perçu (support végétal); M : moyenne ; DS : déviation standard. En gras : résultat statistiquement significatif. 


\section{Discussion}

Ce travail a permis d'identifier les potentialités aussi bien des végétaux que de l'art sur les processus psychologiques des patients atteints de MAMA qui fréquentent ce jardin. Ainsi, il paraît pertinent et profitable de les encourager à s'y rendre lors de promenades accompagnées. Outre l'impact potentiellement bénéfique sur leurs processus mnésiques, les éléments d'aménagement de ce jardin, qu'ils soient naturels ou artistiques, sont propices aux émotions positives de joie et de surprise et constituent de véritables supports à l'imagination et aux réflexions.

Au regard des résultats qualitatifs, les œuvres d'art perçues semblent détenir un fort potentiel d'évocation mnésique. L'art permet de faire ressurgir des connaissances sémantiques liées à une expérience épisodique que le patient partage avec la psychologue de façon détaillée. Les références socio-culturelles exprimées se situent en effet au carrefour de cette épisodicité et de leur savoir sémantique en lien à la culture. Les résultats quantitatifs objectivent aussi ces intuitions : sur le versant émotionnel, car l'art suscite plus l'expression de la joie et de la surprise que les végétaux. Mais aussi, devant une œuvre, leurs connaissances sémantiques sont plus souvent réactivées et manifestées dans le dialogue que devant les végétaux.

Face à ces résultats, nous nous demandons pourquoi la perception d'œuvres d'art stimule-telle plus souvent ces fonctions psychologiques fragilisées par la MAMA que les végétaux?

La contemplation d'œuvres d'art, même à des stades avancés de la maladie, semble être, comme chez le tout venant, une réelle activité qui stimule de nombreuses facultés psychologiques [12-14], et cela, sans apparente mise en échec ressentie. La perception d'objets artistiques, figuratifs ou abstraits, favorise des effets contemplatifs et l'imagination [14] qui s'opèrent lors d'une tâche d'attention soutenue. Les sculptures sont riches car elles représentent un support multi-sensoriel que les patients peuvent non seulement voir mais aussi toucher. Ils peuvent donc être amenés à décrire les composants ou les caractéristiques sensorielles des œuvres. Une sculpture s'appréhende par le mouvement et la déambulation du spectateur pour l'explorer dans sa globalité en raison de ses caractéristiques tridimensionnelles [24]. Le besoin de déambuler que ressentent certains patients atteints de MAMA [25] pourrait, autour des sculptures, être satisfait en étant associé à un éventuel sentiment de surprise engendré par la découverte de certaines faces cachées de la sculpture lors du premier contact visuel.

Bien que ce travail mériterait un approfondissement sur un échantillon plus important tout en tenant compte de certains paramètres pouvant intervenir dans les résultats (e.g., humeur et manifestations émotionnelles avant la promenade), l'ensemble de ces observations nous invite à penser qu'insérer une dimension artistique dans l'aménagement de tels jardins permet aux patients, au-delà des bénéfices cités, de maintenir le lien qu'ils entretiennent avec la culture. Il serait intéressant de prolonger ces travaux afin de déterminer les caractéristiques spécifiques (de formes, de matières...) des composants du jardin (végétaux, œuvres d'art,...) et leur aménagement particulier qui apportent des bénéfices cognitifs et psycho-sociaux aux patients atteints de MAMA. 


\section{Conclusion}

Les patients de notre étude présentent des performances typiques aux tests neuropsychologiques des personnes souffrant de MA, en particulier aux épreuves relatives à la mémoire. Une promenade accompagnée dans cet environnement est une situation de communication plus naturelle que la situation de test. Cette tâche écologique permet d'évaluer de façon moins explicite et potentiellement moins anxiogène, leurs performances dans les mêmes domaines psychologiques qu'une évaluation standard de leurs fonctions cognitives, tout en faisant émerger leurs états émotionnels. C'est ainsi que grâce au jardin thérapeutique, les troubles mnésiques et communicationnels peuvent ne plus être étudiés seulement d'une manière abstraite par des investigations psychométriques, mais ont intérêt à être rapportés à un contexte, tel que le jardin, lieu qui appartient à toutes les cultures façonnant les perceptions de la vie quotidienne. L'analyse des dialogues [15] fait en effet ressortir que la fréquentation du jardin mobilise un système unifié de fonctions psychologiques qui donne accès à différents processus mnésiques et émotionnels suscités par les éléments du jardin. L'exploration en profondeur de l'expérience vécue par le patient qui utilise le jardin, établit des relations spécifiques entre jardin, art, cognition, émotions et langage. On observe que la mise en situation d'interactions répétées éveille aussi la mémoire des relations sociales et met en évidence le partage des appréciations esthétiques. Cette méthode a permis également de mettre en exergue d'autres processus mentaux en lien avec la perception des composants naturels et culturels du jardin, lesquels constituent de véritables supports à l'imagination et aux réflexions introspectives. Cette étude exploratoire, prolongée sur un échantillon conséquent, permet déjà d'envisager des retombées d'ordre thérapeutique en terme de soins éco-psychosociaux pour les patients atteints de maladie d'Alzheimer.

\section{Liens d'intérêts}

Les auteurs déclarent ne pas avoir de liens d'intérêts.

\section{Remerciements}

Nos remerciements vont à la Fondation Lemarchand qui nous a soutenu financièrement pour mener cette recherche.

\section{Références}

[1] Amieva H, Belliard S, Salmon E. Les démences aspects cliniques, neuropsychologiques, physiopathologiques et thérapeutiques. Paris : De Boeck-Solal, 2014.

[2] American Psychiatric Association. Diagnostic and statistical manual of mental disorders. Text Revision 4th ed. Washington : American Psychiatric Association, 2000.

[3] Plan maladies neuro-dégénératives 2014-2019 http://socialsante.gouv.fr/IMG/pdf/Plan_maladies_neuro_degeneratives_def.pdf

[4] Plan Alzheimer 2008-2012 http://www.cnsa.fr/documentation/plan alzheimer 2008-2012-2.pdf

[5] Cohen-Mansfield J, Werner P. Effets d'un jardin thérapeutique sur les troubles du comportement et de l'humeur de résidents de maisons de retraite présentant une déambulation. Maladie d'Alzheimer 1997 ; 4 :199-201. 
[6] Namazi KH, Johnson BD. Pertinent autonomy for residents with dementia. Modifications of the physical environments to enhance independence. Am J Alzheimers Care Relat Disord Res $1992 ; 7$ : $16-21$.

[7] Ousset PJ, Nouhashemi F, Albarede JL et al. Therapeutic gardens, Arch Gerontol Geriatr 1998 ; $26: 369-72$.

[8] Zeisel J, Silverstein N, Hyde J, et al. Environmental correlates to behavioral outcomes in Alzheimer's special care units, Gerontologist $2003 ; 34: 697-711$.

[9] Cohen-Mansfield J, Libin A, Marx M. Non pharmacological treatment of agitation: a controlled trial of systematic individualized intervention. Journals of Gerontology: Medical sciences 2007 ; 62(8) : 908-16.

[10] Zeisel J. I'm Still Here. London : Piatkus, 2010.

[11] Platel H. Art-thérapie et démences : apports de la neuropsychologie. Rev Neuropsychol $2011 ; 3$ : 205-6.

[12] Changeux JP. Raison et plaisir. Paris : Odile Jacob, 1994.

[13] Changeux JP. Le cerveau et l'art. Paris : De Vive Voix, 2010.

[14] Borillo M (Dir). Dans l'atelier de l'art. Expériences cognitives. Seyssel : Champ Vallon, 2010.

[15] Trognon A, Batt M, Bromberg M et al. Les formes logiques de la parole-en- interaction quelques bases de la logique interlocutoire. In : Castel P, Lacassagne MF, Salès-Wuillemin E, eds. Psychologie sociale de la communication 2. Paris : De Boeck, 2011 : 327-46.

[16] Rivasseau-Jonveaux T, Batt M, Fescharek R et al. Healing gardens and cognitive behavioral units in the Management of Alzheimer's disease patients: the Nancy experience. J Alzheimers Dis 2013 ; 34(1) : 325-338.

[17] Rivasseau-Jonveaux T, Pop A, Fescharek R et al. Les jardins thérapeutiques : recommandations et critères de conception. Geriatr Psychol Neuropsychiatr Vieil $2012 ; 10(3): 245-53$.

[18] Poitrenaud J. L'évaluation du niveau socio-culturel. In : Hugonot-Diener L, ed. La consultation en Gériatrie. Paris : Masson, 2001 : 8-9.

[19] Folstein MF, Folstein SE, McHugh PR. « Mini-mental state». A practical method for grading the cognitive state of patients for the clinicial. J Psychiatr Res $1975 ; 12$ : 189-98.

[20] Dubois B, Touchon, J, Portet F et al. Les 5 mots : un test simple et sensible pour le diagnostic de la maladie d'Alzheimer, Persse Méd 2002, $31:$ 1696-99.

[21] Dubois B, Slachevsky A, Litvan I, Pillon B. The FAB : A frontal assessment battery at bedside. Neurology 2000, 55(11) : 1621-6.

[22] McKhann G, Drachman D, Folstein M et al. Clinical diagnosis of Alzheimer's disease: report of the NINCDS-ADRDA Work Group under the auspices of Department of Health and Human Services Task Force on Alzheimer's Disease. Neurology 1984 ; 34(7): 939-44.

[23] Bottineau D. OUPS! Les émotimots, les petits mots des émotions : des acteurs majeurs de la cognition verbale interactive. Langue française $2013 ; 180(4): 99-112$.

[24] Bernard M, Gefen A, Talon-Hugon C. (Dirs) Arts et émotions: Dictionnaire. Paris : Armand Colin, 2016.

[25] Cooper-Marcus C C, Barnes M. Healing Gardens: Therapeutic benefits and design recommendations. John Wiley \& Sons, 1999. 